\title{
Les peptides du lait à activité physiologique III. Peptides du lait à effet cardiovasculaire : activités antithrombotique et antihypertensive
}

\author{
JL Maubois ${ }^{1}$, J Léonil ${ }^{1}, \mathrm{R}$ Trouvé 2 , S Bouhallab 1 \\ 1 INRA, laboratoire de recherches de technologie laitière, \\ 65 , rue de Saint-Brieuc, 35042 Rennes cedex \\ ${ }^{2}$ CNTS, 3 avenue des tropiques, BP 100, 91943 Les Ulis cedex, France
}

\begin{abstract}
Résumé - Les similarités fonctionnelles entre la coagulation du lait et celle du sang, ainsi que les homologies de séquences existant entre la chaîne $\gamma$ du fibrinogène et la caséine $\kappa$ du lait, ont été à la base de la démarche adoptée par le groupe de Jollès pour démontrer l'activité cardiovasculaire d'une famille de peptides issus de cette protéine du lait. Ces peptides localisés dans le segment glycomacropeptidique de la caséine $\kappa$, sont capables, non seulement d'inhiber l'agrégation plaquettaire mais aussi de se lier avec les récepteurs spécifiques apparaissant à la surface de ces éléments figurés du sang et donc, de prévenir la formation du thrombus.

L'activité antiagrégative est nettement renforcée par la présence d'un résidu Lys; ainsi, la séquence 112-116 semble 200 fois plus active que le peptide 113-116. Les résultats obtenus par notre groupe à partir de peptides résultant de l'hydrolyse trypsique du GMP ont confirmé et précisé ceux du groupe de Jollès.

L'hydrolyse de l'angiotensine I par l'enzyme de conversion de l'angiotensine (ACE) est l'étape clé de la régulation physiologique de la tension artérielle. Aussi a-t-on recherché des séquences peptidiques à même d'inhiber l'action de l'ACE. De telles molécules ont été localisées dans la séquence de la caséine $\beta$.

L'activité biologique des protéines laitières semble de plus en plus plausible au vu des résultats acquis depuis une dizaine d'années qui, outre la démonstration d'activités spécifiques in vivo et in vitro, indiquent la multifonctionnalité de certaines séquences et la conservation de ces séquences dans la plupart des espèces de mammifères.
\end{abstract}

lait / caséine / peptide / activité cardiovasculaire / activité biologique

Summary - Milk peptides with cardiovascular activity: antithrombotic and antihypertensive activities. Functional similarities between milk and blood coagulation as well as sequence homologies existing in fibrinogen $\gamma$ chain and $k$ casein were the basis for the approach which the Jollès group adopted to demonstrate the cardiovascular activity of a peptide family issued from this milk protein. These peptides are located in the glycomacropeptide segment of $k$ casein. They are able not only to inhibit platelet aggregation but also to combine with receptor sites and consequently to prevent fibrinogen binding with blood platelets (antitrhrombotic activity). In vitro antiaggregative activity is reinforced by the presence of a Lys residue in the sequence; therefore, the 112-116 peptide resulting from tryptic hydrolysis of GMP seems to be 200-fold more active than the 113-116 sequence. These recent findings by our team have confirmed those of the Jollès group. Hydrolysis of angiotensin I by angiotensin converting enzyme (ACE) is the key step in the physiological regulation of blood pressure. An investigation was therefore carried out on peptidic sequences able to block $A C E$ action with a view to finding therapeutic natural substances acting against blood hypertension. Such peptides are located in $\beta$ casein, and some, such as the 43-52 human $\beta$ casein sequence, seem to be very efficient. The biological activity of milk proteins seems to be an increasingly plau- 
sible possibility, given the results acquired during the last decade, which besides the demonstration of specific activities in vitro and in vivo, indicate the multifunctionality of certain sequences and the conservation of specific sequences in milk proteins produced by many mammalian species.

milk / casein / peptide / biological activity / cardiovascular activity

\section{INTRODUCTION}

Trois stratégies de recherches sont envisageables (Meisel et al, 1989) pour l'étude et la caractérisation de séquences peptidiques ayant une activité biologique :

- recherche de protéines dont les séquences d'acides aminés sont similaires à celles de protéines actives connues. Ces séquences sont ensuite synthétisées et évaluées par leurs propriétés bioactives;

- isolation et caractérisation de peptides actifs à partir d'hydrolysats protéiques in vitro;

- isolation et caractérisation de peptides actifs issus de la digestion gastrointestinale chez l'animal.

Ces 3 approches ont été suivies dans le cas des protéines laitières où de multiples activités ont été mises en évidence (Maubois et Léonil, 1989; Fiat et Jollès, 1989).

Dans le cadre de ces journées, notre propos sera limité à la synthèse des derniers travaux réalisés sur les activités cardiovasculaires mises en évidence dans les composants protéiques du lait.

Les maladies cardiovasculaires constituent la plus grande cause de mortalité de la population adulte dans les pays industrialisés. La formation d'un thrombus (caillot) au niveau des vaisseaux sanguins (Zucker, 1980) ou encore la vasoconstriction (Wyvratt et Patchett, 1985) sont parmi les événements les plus fréquents à l'origine des dysfonctionnements du système vasculaire.
Les étapes clés des mécanismes intervenant dans ces dérèglements du système vasculaire ont été récemment identifiées. II a donc pu être imaginé et expérimenté des stratégies mettant en œuvre des molécules ayant une activité d'antagoniste spécifique et bloquant ainsi le déroulement de ces mécanismes. Le fait de disposer de la connaissance complète des séquences primaires des protéines laitières a représenté un outil précieux dans la recherche de dérivés peptidiques à même de "leurrer" des récepteurs cellulaires ou d'inhiber l'action d'enzymes par blocage de leurs sites actifs.

\section{ACTIVITÉ ANTITHROMBOTIQUE}

La formation des thrombus dans les vaisseaux sanguins découle d'une cascade extrêmement complexe de réactions (Zucker, 1980). Parmi la multitude des événements qui entrent en jeu, celui qui apparaît être le plus critique est la liaison du fibrinogène aux récepteurs plaquettaires (Shebuski et al, 1989). Après activation par des agonistes physiologiques (ADP, thrombine, etc.) les plaquettes fixent le fibrinogène ainsi que d'autres protéines, telles que la fibronectine et le facteur de von Willebrand. La liaison de ces protéines, dites adhésives, se fait au niveau de récepteurs glycoprotéiques GPIIb-GPIIla développés à la surface des plaquettes activées (Plow et al, 1985).

Des anomalies héréditaires de ces récepteurs ou la présence d'anticorps contre 
GPIIb-GPIIla conduisent à une tendance sévère au saignement. Les expériences réalisées chez l'animal ont montré que les anticorps anti-GPIIb-GPIIla sont en effet de puissants agents antithrombotiques et antihémostase. Cette voie immunologique a d'ailleurs été proposée pour des applications cliniques (Coller et al, 1988).

Les séquences peptidiques du fibrinogène impliquées dans l'interaction avec les récepteurs des cellules plaquettaires ont été identifiées. II s'agit du tétrapeptide RGD/X (fragment 572-575) de la chaîne $\alpha$ et la séquence 400-411 de la chaîne $\gamma$ du fibrinogène (Shebuski et al, 1989). Les expériences réalisées tant in vitro qu'in vivo ont montré que des analogues synthétiques de ces séquences étaient capables d'inhiber l'agrégation plaquettaire et donc la formation du thrombus (Kloczewiak et al, 1984; Gartner et Bennett, 1985; Cadroy et al, 1989; Shebuski et al, 1989).

Se basant sur la similarité des phénomènes de coagulation du sang et du lait, Jollès et al (1986) puis Drouet et al (1990) ont recherché l'existence de séquences inhibitrices au sein des protéines du lait.

Deux séquences analogues à celles contenues dans la molécule du fibrinogène ont été identifiées dans les protéines lai- tières. II s'agit d'une part, d'un analogue du tétrapeptide RGD/X du fibrinogène $\alpha$, localisé dans la région 39-42 de la lactotransferrine humaine. Les tests réalisés, après synthèse chimique de ce fragment, ont montré que cette séquence a une activité antiplaquettaire in vitro et antithrombotique in vivo. De plus, cette séquence coinjectée en présence du fragment synthétique du fibrinogène $\alpha$ a un effet potentialisateur chez le cobaye et surtout chez le rat. D'autre part, une région analogue au fragment 400-411 (partie C-terminale) du fibrinogène $\gamma$ a été identifiée dans la partie $\mathrm{N}$-terminale du caséinoglycomacropeptide (CMP) issu de l'hydrolyse par la chymosine de la caséine $\kappa$ bovine. Divers peptides naturels (hydrolyse trypsique du CMP) ou synthétiques de cette région (106-116; 106-112; 113-116) (tableau I) ont été testés pour leur capacité à inhiber la fixation du fibrinogène et l'agrégation des plaquettes. Le fragment 106-116 naturel s'est révélé être le plus actif dans cette étude (Jollès et al, 1986).

Dans la même ligne de recherche, le pentapeptide 112-116 obtenu par hydrolyse trypsique du CMP (Léonil et Mollé, 1990) a été testé pour ses capacités à inhiber l'agrégation des plaquettes in vitro (Léonil et al, résultats non publiés). Les ré-

Tableau I. Structure primaire des peptides des la région N-terminale du CMP dérivé de la caséine $\kappa$ bovine, et du fragment $\mathrm{C}$-terminal de la chaîne $\gamma$ du fibrinogène.

Primary sequence of peptides from the $\mathrm{N}$-terminal region of bovine glycomacropeptide and from the c-terminal region of the $\gamma$ chain of fibrinogen.

KCN (106-116)

$(106-112)$

(113-116)

$(112-116)$
$\mathrm{H}_{2} \mathrm{~N}-\mathrm{M}-\mathrm{A}-\mathrm{I}-\mathrm{P}-\mathrm{P}-\mathrm{K}-\mathrm{K}-\mathrm{N}-\mathrm{Q}-\mathrm{D}-\mathrm{K}-\mathrm{COOH}$

$\mathrm{H}_{2} \mathrm{~N}-\mathrm{M}-\mathrm{A}-\mathrm{I}-\mathrm{P}-\mathrm{P}-\mathrm{K}-\mathrm{K}-\mathrm{COOH}$

$\mathrm{H}_{2} \mathrm{~N}-\mathrm{N}-\mathrm{Q}-\mathrm{D}-\mathrm{K}-\mathrm{COOH}$

$\mathrm{H}_{2} \mathrm{~N}-\mathrm{K}-\mathrm{N}-\mathrm{Q}-\mathrm{D}-\mathrm{K}-\mathrm{COOH}$

Fibrinogène $\gamma$ : (400-411)

$\mathrm{H}_{2} \mathrm{~N}-\mathrm{H}-\mathrm{H}-\mathrm{L}-\mathrm{G}-\mathrm{G}-\mathrm{A}-\mathrm{K}-\mathrm{Q}-\mathrm{A}-\mathrm{G}-\mathrm{D}-\mathrm{V}-\mathrm{COOH}$ 
sultats préliminaires obtenus ont montré que ce peptide était respectivement 30 fois et 200 fois plus actif que les peptides 106-116 et 113-116 décrits par Jollès et al (1986) (tableau II). Dans la mesure où les résultats issus de ces 2 études sont comparables, la présence ou l'absence du résidu lysine (position 112) pourrait expliquer ces différences d'activité constatées. En effet, Kloczewiak et al (1984) ont montré que le résidu Lys 406 du segment 400-411 du fibrinogène $\gamma$ était impliqué dans l'interaction de cette molécule avec les récepteurs plaquettaires.

Les segments peptidiques issus de I'hydrolyse enzymatique ménagée de la portion N-terminale du CMP constituent donc une famille de molécules capables d'inhiber l'agrégation plaquettaire.

\section{ACTIVITÉ ANTIHYPERTENSIVE}

L'hydrolyse de l'angiotensine, molécule présente dans le plasma sanguin par l'enzyme de conversion de l'angiotensine (ACE : E.C.3.4.15.1) est certainement l'étape clé dans la cascade d'événements biochimiques conduisant à la constriction des artérioles et donc, à la remontée rapide de la pression artérielle. Le clivage par l'ACE des 2 résidus $\mathrm{C}$ terminaux (His-
Leu) sous forme de dipeptide transforme en effet l'angiotensine I en un puissant vasoconstricteur : I'angiotensine II.

Ce rôle clé de l'ACE, sa localisation dans de nombreux tissus y compris dans les cellules intestinales (Stevens et al, 1988; Duggan et al, 1989) justifient qu'elle ait été choisie comme cible privilégiée des agents thérapeutiques à même d'intervenir contre l'hypertension. Les permières molécules actives qui aient été identifiées étaient des petits peptides présents dans le venin d'un serpent brésilien. Ces peptides inhibent l'action de l'ACE en bloquant son site actif. Ils contiennent un enchaînement particulier d'acides aminés dont une proline en position C-terminale.

En raison de la richesse élevée des caséines en résidus proline, Maruyama et al (1985) ont recherché de tels inhibiteurs dans les hydrolysats enzymatiques des caséines bovines. Des séquences des caséines $\alpha_{s}$ et $\beta$ capables d'inhiber l'ACE in vitro ont ainsi été identifiées. Plus récemment, Karaki et al (1990) ont rapporté que ces mêmes séquences avaient un effet anti-hypertensif chez les rats rendus artificiellement hypertensifs.

Dans cette même optique, en se basant sur les données structurales des peptides inhibiteurs de l'ACE, Kohmura et al (1989, 1990 ) ont reproduit par synthèse chimique

Tableau II. Concentration des peptides inhibant 50\% de l'agrégation des plaquettes activées en présence de l'ADP

Peptide concentration inhibiting $50 \%$ of the aggregation of ADP-activated platelets.

Séquences $\quad 1 C_{50}(\mu \mathrm{mol} / \mathrm{l})$

$\begin{array}{lr}\text { KCN }(112-116) & 2 \\ \operatorname{KCN}(106-116)^{*} & 60 \\ \mathrm{KCN}(113-116)^{*} & 400\end{array}$

\footnotetext{
${ }^{*}$ Résultats publiés par Jollès et al (1986).

"Results published by Jollès et al,1986.
} 
des séquences des caséines $\beta$ et $\kappa$ humaines possédant un résidu proline en C-terminal.

Parmi les 92 séquences synthétisées dont 69 de la caséine $\beta$ et 23 de la caséine $\kappa, 4$ possèdent une forte activité inhibitrice de I'ACE proche de celle décrite dans le venin de serpent (tableau III). La séquence 43-52 de la caséine $\beta$, n'est que 4 fois moins active que l'agent de référence $S Q$ 20881.

\section{PEPTIDES OPIOIIDES ET EFFET CARDIOVASCULAIRE}

Les nombreuses actions pharmacologiques des peptides opioïdes ont été récemment revues par Ramabadran et Bansinath (1989). L'administration intracérébrale ou intraveineuse de ces molécules conduit à de puissants effets sur le système cardiovasculaire. Chez des rats normotensifs anesthésiés, l'injection intracérébroventriculaire ou intraveineuse de la $\beta$ casomorphine (1-4) amidée produit de l'hypertension et de la bradycardie. Ces phénomènes sont réversés par la naloxone, antagoniste spécifique des opiacés (Wei et al, 1980). En revanche, lorsque I'injection intraventriculaire de la $\beta$-casomorphine (14) amidée était effectuée chez des rats hypertensifs, une augmentation de la pression sanguine et une accélération du rythme cardiaque étaient observées. Par ailleurs, Liebmann et al (1986) ont rapporté l'effet de faibles concentrations de $\beta$ casomorphine (1-5) sur les membranes du muscle cardiaque chez le cobaye. Leurs résultats suggèrent qu'une action sur la pompe à sodium pourrait être à l'origine de l'effet de cette substance sur le potentiel d'action du myocarde. Sur un autre plan, il est connu que les opioïdes endogènes sont des inhibiteurs de l'ACE (Holaday, 1983). Bien que ce phénomène n'ait pas été démontré pour les exorphines, ceci laisse à penser que ces molécules agissent par différents mécanismes sur le système cardiovasculaire.

\section{CONCLUSIONS}

Outre leur qualité nutritionnelle parfaitement adaptée à la satisfaction des besoins de l'être humain, les protéines du lait sont des vecteurs d'activités biologiques. Une

Tableau III. Concentration des peptides inhibant $50 \%$ de l'activité de l'enzyme de conversion de l'angiotensine (ACE). * Produit de référence.

(1) Maruyama et al (1987); (2) Kohmura et al (1990); (3) Kohmura et al (1989)

Peptide concentration inhibiting $50 \%$ of angiotensin converting enzyme activity.

\begin{tabular}{clll}
\hline Séquences & Origine & IC50 $(\mu \mathrm{mol} / \mathrm{l})$ & Référence \\
\hline $177-183$ & Caséine $\beta$ bovine & 15 & 1 \\
$23-27$ & Caséine $\alpha_{\mathrm{S} 1 \text { bovine }}$ & 6 & 1 \\
$18-20$ & Caséine $\kappa$ humaine & 3,5 & 2 \\
$124-129$ & Caséine $\beta$ humaine & 2,9 & 3 \\
$63-65$ & Caséine $\kappa$ humaine & 2,2 & 2 \\
$43-52$ & Caséine $\beta$ humaine & 1,4 & 3 \\
SQ 20881* & Venin de serpent & 0,56 & 3 \\
\hline
\end{tabular}


telle conclusion s'impose, sans conteste, au vu des résultats accumulés depuis le travail de pionnier réalisé par Brantl et al (1979) sur les activités opioïdes de plusieurs segments de la caséine $\beta$. Mais pour intéressantes qu'elles soient, les connaissances accumulées depuis une dizaine d'années sont encore par trop fragmentaires, par trop basées sur des tests in vitro pour affirmer avec certitude que ces activités sont exercées après chaque ingestion de produits laitiers dans les organismes humains. De nombreuses études doivent encore être menées pour confirmer ce rôle in vivo des protéines laitières mais, outre les résultats déjà acquis, 2 autres observations sont en faveur d'un rôle biologique de certaines séquences précises :

- ces enchaînements sont conservés dans les diverses espèces de mammifères;

- ces peptides sont multifonctionnels ; une même séquence intervient au niveau de plusieurs activités; par exemple, la $\beta$ casomorphine cumulerait des activités morphinomimétique et immunostimulante, la séquence 177-183 de la caséine $\beta$ associerait des activités immunostimulante et antihypertensive.

Parmi ces activités biologiques, celles agissant au niveau cardiovasculaire ont été particulièrement bien établies in vitro. Mais les mécanismes permettant le transfert des séquences à travers la paroi intestinale ne sont pas, ou peu connus. Leur détermination permettrait d'envisager la forme sous laquelle pourrait être apporté(s) le ou les peptides actif(s).

\section{RÉFÉRENCES}

Brantl V, Teschemacher H, Henschen A, Lottspeich $F$ (1979) Novel opioid peptides derived from casein ( $\beta$-casomorphins). HoppeSeyler's Z Physiol Chem 360, 1211-1216
Cadroy Y, Houghten RA, Hanson SR (1989) RGDV peptide selectively inhibits plateletdependent thrombus formation in vivo. Studies using a baboon model. J Clin Invest 84 , 939-944

Coller BS, Scudder LE, Berger HJ, Iulicci JD (1988) Inhibition of human platelet function in vivo with a monoclonal antibody: with observations on the newly dead as experimental subject. Ann Int Med 109, 635-638

Drouet L, Bal dit Sollier C, Cisse M, Pignaud G, Mazoyer $E$, Fiat AM, Jollès $P$, Caen JP (1990) The antithrombotic effect of KRDS, a lactotransferrin peptide, compared with RGDS. Nouv Rev Fr Hemato/32, 59-62

Duggan KA, Mendelsohn FAO, Levens NR (1989) Angiotensin receptors and angiotensin I-converting-enzyme in rat intestine. Am J Physiol 257, G504-G510

Fiat AM, Jollès $P$ (1989) Caseins of various origins and biologically active casein peptides and disaccharides: structural and physiological aspects. Mol Cell Biochem 87, 5-30

Gartner TK, Bennett JS (1985) The tetrapeptide analogue of the cell attachment site of fibronectin inhibits platelet aggregation and fibrinogen binding to actived platelets. I Biol Chem 260, 11891-11894

Holaday JW (1983) Cardiovascular effects of endogenous opiate systems. Annu Rev Pharmacol Toxicol 23, 541-594

Jollès $P$, Levy-Toledano $S$, Fiat $A M$, Soria $C$, Gillessen D, Thomaidis A, Dunn FW, Caen JP (1986) Analogy between fibrinogen and casein. Effect of an undecapeptide isolated from $\kappa$-casein on platelet function. Eur $J$ Biochem 158, 379-382

Karaki H, Doi K, Sugano S, Uchiwa H, Sugai R, Murakami U, Takemoto S (1990) Antihypertensive effect of tryptic hydrolysate of milk casein in spontaneously hypertensive rats. Comp Biochem Physiol 96C, 367-371

Kloczewiak M, Timmons S, Lukas TJ, Hawiger J (1984) Platelet receptor recognition site on human fibrinogen. Synthesis and structurefunction relationship of peptides corresponding to the carboxy-terminal segment of the $\gamma$-chain. Biochemistry 23, 1767-1774

Kohmura M, Nio N, Kubo K, Minoshima Y, Munekata $E$, Ariyoshi $Y$ (1989) Inhibition of angiotensin-converting-enzyme by synthetic peptides of human $\beta$-casein. Agric Biol Chem $53,2107-2114$ 
Kohmura M, Nio N, Ariyoshi Y (1990) Inhibition of angiotensin-converting-enzyme by synthetic peptides of human $\kappa$-casein. Agric Biol Chem 54, 835-836

Léonil J, Mollé D (1990) Liberation of tryptic fragments from caseinomacropeptide of bovine $\kappa$-casein involved in platelet function. Kinetic study. Biochem J 271, 247-252

Liebmann C, Barth A, Neubert K, Mentz P, Hoffmann $S$ (1986) Effects of $\beta$-casomorphin on ${ }^{3} \mathrm{H}$-ouabain binding to guinea-pig heart membranes. Pharmazie 41, 670-671

Maruyama S, Nakagomi K, Tomizuka N, Suzuki $\mathrm{H}$ (1985) Angiotensin I-converting-enzyme inhibitor derived from an enzymatic hydrolysate of casein. II-Isolation and bradykininpotentiating activity on the uterus and the ileum of rats. Agric Biol Chem 49,1405-1409

Maruyama S, Mitachi H, Tanaka H, Tomizuka N, Suzuki $H$ (1987) Studies on the active site and antihypertensive activity of angiotensin Iconverting-enzyme inhibitors derived from casein. Agric Biol Chem 51, 1581-1586

Maubois JL, Léonil J (1989) Peptides du lait à activité biologique. Lait 69, 245-269

Meisel H, Frister H, Schlimme E (1989) Biologically active peptides in milk proteins. $Z$ Ernahrungswiss 28, 267-278

Plow EF, McEver RP, Coller BS, Woods VL, Marguerie GA, Ginsberg MH (1985) Related binding mechanims for fibrinogen, fibronectin, von Willebrand factor, and thrombospondin on thrombin-stimulated human platelets. Blood 66, 724-727

Ramabadran K, Bansinath M (1989) Pharmacology of $\beta$-casomorphins, opioid peptides derived from milk protein. Asia Pacific J Pharmacol 4, 45-58

Shebuski RJ, Berry DE, Bennett DB, Romoff T, Storer BL, Ali F, Samanen J (1989) Demonstration of Ac-Arg-Gly-Asp-Ser- $\mathrm{NH}_{2}$ as an antiaggregatory agent in the dog by intracoronary administration. Thromb Haemostasis 61, 183-188

Stevens BR, Fernandez A, Kneer C, Cerda JJ, Phillips MI, Woodward ER (1988) Human intestinal brush border angiotensin-convertingenzyme activity and its inhibition by antihypertensive ramipril. Gastroenterology 94, 942-947

Wei ET, Lee A, Chang JK (1980) Cardiovascular effects of peptides related to the enkephalins and $\beta$-casomorphin. Life Sci 26, 15171522

Wyvratt MJ, Patchett AA (1985) Recent developments in the design of angiotensinconverting-enzyme inhibitors. Med Res Rev $5,483-531$

Zucker M (1980) Plaquettes sanguines et coagulation. Pour Sci 34, 37-47 